\title{
Radial and vertical angular momentum transport in protostellar discs
}

\author{
Raquel Salmeron $^{1}$ • Arieh Königl • Mark Wardle
}

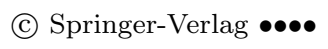

\begin{abstract}
Angular momentum in protostellar discs can be transported either radially, through turbulence induced by the magnetorotational instability (MRI), or vertically, through the torque exerted by a large-scale magnetic field. We present a model of steady-state discs where these two mechanisms operate at the same radius and derive approximate criteria for their occurrence in an ambipolar diffusion dominated disc. We obtain "weak field" solutions - which we associate with the MRI channel modes in a stratified disc - and transform them into accretion solutions with predominantly radial angular-momentum transport by implementing a turbulent-stress prescription based on published results of numerical simulations. We also analyze "intermediate field strength" solutions in which both radial and vertical transport operate at the same radial location. Our results suggest, however, that this overlap is unlikely to occur in real discs.
\end{abstract}

Keywords accretion, accretion discs - MHD — stars: formation — ISM: jets and outflows.

\section{Introduction}

Angular momentum in protostellar discs can be transported either radially, through turbulent viscosity induced by the magnetorotational instability (MRI; e.g.

Raquel Salmeron

Research School of Astronomy and Astrophysics and Research School of Earth Sciences, The Australian National Universty.

Arieh Königl

Department of Astronomy \& Astrophysics, The University of Chicago

Mark Wardle

Physics Department, Macquarie University

${ }^{1}$ Previous address: Department of Astronomy \& Astrophysics, The University of Chicago.
Balbus \& Hawley 1998); or vertically, by centrifugally driven winds (e.g. Königl \& Pudritz 2000). It is likely, in fact, that both forms of transport play a role in real discs ${ }^{1}$ Quasi-steady disc models that incorporate the two mechanisms have already been attempted (e.g. Lovelace et al. 1994; Casse \& Ferreira 2000; Ogilvie \& Livio 2001), but these studies treated the radial transport using prescriptions that do not account for its origin in MRI-induced turbulence. A more refined approach seems to be needed, however, as solutions in which a wind carries away all the excess angular momentum of the fluid are not susceptible to sustain MRI-unstable modes (Wardle \& Königl 1993, hereafter WK93). Our prescription to incorporate radial transport in our wind-driving models uses the fact that the growth (and structure) of the MRI is dependent on the local strength of the magnetic field, which we measure by $a$, the ratio of the Alfvén speed to the isothermal sound speed (see section 2). MRI modes are expected to grow when $a \ll 1$ (provided that the gas is sufficiently coupled to the field), but they are suppressed when $a$ is comparatively large $(\gtrsim 1)$. Since $a\left(\propto \rho^{-0.5}\right)$ increases with height, this suggest that radial and vertical angular momentum transport could in principle operate at the same radius, with the MRI acting in a finite section next to the midplane, and outflows dominating at higher $z$.

Since protostellar discs are weakly ionized over most of their extent, it is essential to incorporate the effect of finite magnetic diffusivity in our models. This is done in our general scheme by employing a tensorconductivity, obtained via a realistic vertical ionization structure. This formulation naturally incorporates the

${ }^{1}$ Note that vertical angular-momentum transport could also occur via other mechanisms, not considered here; such us magnetic braking (e.g. Krasnopolsky \& Königl 2002), "failed" winds or non-steady phenomena. 
three relevant magnetic diffusion mechanisms (i.e. ambipolar, Hall and Ohmic). The illustrative examples presented here, however, assume that ambipolar diffusion dominates over the entire section of the disc and (except in section 44), take the magnetic coupling to be constant with height. This is useful in order to apply the analytic results obtained by WK93 for this case to our prescription for MRI-induced turbulent transport in a wind-driving disc model. We refer the reader to Salmeron, Königl \& Wardle 2007 (hereafter SKW07) for full details of the study. Section 2 presents the wind-driving model. Section 3 outlines our prescription for MRI-induced radial transport and illustrates it with a solution with a predominantly radial angularmomentum transport. Section 4 discusses a representative solution that incorporates radial and vertical transport and our findings are summarised in section 5 .

\section{Centrifugally driven winds}

Bipolar outflows are commonly associated with protostellar accretion discs, as well as other astronomical accreting sources. Their ubiquity suggests that they play a key role in regulating accretion, a notion supported by the apparent correlation between accretion and outflow signatures in these systems (e.g. Hartigan, Edwards \& Ghandour 1995). It is also generally accepted that these outflows are highly collimated winds, accelerated centrifugally from the surfaces of discs by the mechanism first proposed by Blandford \& Payne (1982, hereafter $\mathrm{BP} 82$ ). According to this picture, disc material is flung out if the open magnetic field lines that thread the disc are sufficiently inclined (i.e. the angle between the line and the disc plane is $<60^{\circ}$ ), initiating an outflow that can potentially reach super Alfvénic speeds.

A key feature of these winds, and one of particular interest in the context of this work, is that they provide an efficient means of extracting the rotational kinetic energy of the disc and carrying away the excess angular momentum of the accreted matter. This is expected to be the dominant angular momentum transport mechanism in discs when the magnetic field is strong (suprathermal) and the MRI is suppressed because the wavelength of the most unstable perturbations exceed the magnetically reduced disc scale height. Wind-driving solutions from strongly magnetised discs have been obtained semi-analytically and numerically (e.g. WK93; Li 1995, 1996; Casse \& Keppens 2002).

In this work we have used the modelling procedure developed by WK93 to obtain radially localised discwind solutions from strongly magnetised discs. This entails solving the conservation (of mass and momentum) and induction equations, as well as Ampère's and
Ohm's laws for a steady-state, isothermal, geometrically thin and nearly Keplerian accretion disc. We neglect all radial derivatives except that of $v_{\phi}$ and use a conductivity tensor (e.g. Wardle 1999) to communicate the effect of the charged species on the neutrals.

These equations are integrated vertically upwards to obtain the disc solution and the location of the critical (sonic) point. This solution is then matched onto a BP82, self-similar wind solution, by imposing the regularity condition at the Alfvén critical point. The relevant model parameters are (1) $a_{0}=v_{\mathrm{A} 0} / c_{\mathrm{s}}$, the midplane (subscript 0 ) ratio of the Alfvén speed to the sound speed, which measures the magnetic field strength; (2) $\eta$, the ratio of the Keplerian rotation time to the neutral-ion momentum-exchange time, which determines the magnetic coupling; (3) $\epsilon \equiv-v_{r 0} / c_{\mathrm{s}}$, the midplane (normalized) inward radial speed, evaluated by imposing the Alfvén critical-point constraint on the wind; (4) $c_{\mathrm{s}} / v_{\mathrm{K}}=h_{\mathrm{T}} / r$, the ratio of the disc tidal scale height to the radius; and (5) $\epsilon_{B} \equiv-c E_{\phi} / c_{\mathrm{s}} B_{z}$, the normalized azimuthal component of the electric field, which is nonzero if the magnetic field lines drift radially (WK93) ${ }^{2}$

The main properties of wind-driving, strongly magnetized $\left(a_{0} \lesssim 1\right)$ discs are analysed in WK93. Fig. 1 of SKW07 shows a solution of this type that matches on to a global BP82 wind solution (see also the pure-wind case of Fig. 2). These solutions satisfy:

$$
(2 \eta)^{-1 / 2} \lesssim a_{0} \lesssim \sqrt{3} \lesssim \epsilon \eta ; \quad \eta>1 .
$$

The first inequality in (1) expresses the requirement that $v_{\phi}<v_{\mathrm{K}}$ in the disc region, the second gives the wind launching condition $\left(B_{r, b} / B_{z}>1 / \sqrt{3}\right.$, where subscript ' $b$ ' denotes the disc surface), the third constrains the base of the wind to lie above the magneticallyreduced density scale height $(h)$ and the fourth is the minimum field-matter coupling condition.

\section{MRI-induced turbulence}

The MRI transports angular momentum radially outwards as fluid elements exchange angular momentum non-locally via the distortion of the magnetic field lines that connect them. Its properties have been extensively investigated in both the linear and nonlinear stages (e.g. Sano \& Stone 2002a,b; Salmeron \& Wardle 2003, 2005). In particular, numerical simulations

\footnotetext{
${ }^{2}$ Setting $\epsilon_{\mathrm{B}}=0$ effectively fixes the value of $B_{r}$ at the disc surface (subscript ' $\mathrm{b}$ '). In a global formulation $B_{r}$ would be determined by the radial distribution of $B_{z}$ (e.g. Ogilvie \& Livio 2001; Krasnopolsky \& Königl 2002).
} 
show that the MRI sustains radial angular-momentum transport when the initial (subscript i) Elsasser number $\Lambda_{\mathrm{i}} \equiv v_{\mathrm{A} 0, \mathrm{i}}^{2} / \eta_{\mathrm{Ohm}} \Omega_{\mathrm{K}}$ is $\gtrsim 1$ (e.g. Sano \& Inutsuka 2001 ; Sano \& Stone 2002a,b), where $\eta_{\mathrm{Ohm}}$ is the Ohmic diffusivity. For the vertical initial field geometry assumed here, it turns out that $\Lambda_{\mathrm{i}}=\eta_{\mathrm{i}}(\mathrm{SKW} 07)$ and the minimum-coupling condition is the same for both forms of angular-momentum transport. So the question we seek to answer is: Assuming that $\eta \gtrsim 1$, which section of the disc (if any) would be unstable to the MRI? The first inequality in (1) can in fact be used

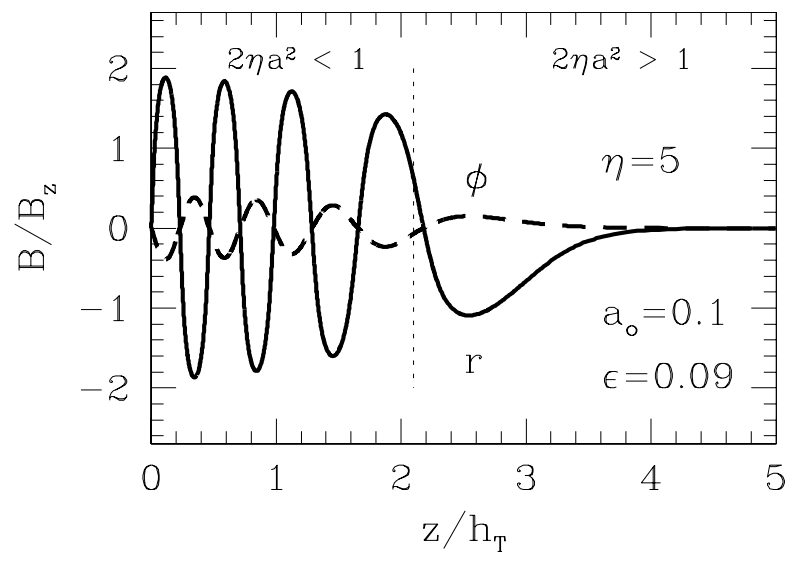

Fig. 1.- Structure of a weakly magnetized disc with vertical magnetic angular-momentum transport in the limit where no wind develops. The radial and vertical components of the magnetic field are indicated by the thick solid and dashed lines, respectively. The vertical dotted line marks the boundary of the MRI-unstable zone (see text).

to derive a lower limit of $a$ for MRI stability. This is the case because when this expression is violated, the surface layers of the disc become super-Keplerian and experience outward streaming motion that is unphysical for a pure wind solution (WK93), but is characteristic of the two-channel mode that underlies the MRI. Moreover, wind-driving disc solutions that satisfy all the constraints in (1) are stable to the fastest growing linear mode of the MRI (WK93), a condition that further supports this choice. The above constraint on $a$ was applied by WK93 at the midplane, since in their solutions the wind carries away the entire excess angular momentum of the gas. Here, however, it is more appropriate to apply it locally [using $a(z) \propto \rho(z)^{-1 / 2}$ ] to differentiate the MRI-unstable section of the disc (where $\left.2 \eta a^{2}<1\right)$ from the region where only vertical angular momentum transport takes place $\left(2 \eta a^{2}>1\right)$.

Fig. 11 shows a solution where $a<(2 \eta)^{-1 / 2}$ over most of the disc vertical extent. Note that no wind develops and thus, angular momentum is transported magnetically between different heights and ultimately carried away radially by the flow. As anticipated, both the radial and azimuthal field components oscillate in the region where $2 \eta a^{2} \lesssim 1$ and the disc sections where $B_{z} \partial B_{\phi} / \partial z<0 \quad(>0)$ lose (gain) angular momentum and have $v_{r}<0 \quad(>0)$. We associate this solution with a two-channel MRI mode for a stratified, non-ideal MHD disc. These modes were analysed by Goodman \& $\mathrm{Xu}$ (1994) in the unstratified, ideal-MHD case. These authors showed that they are exact solutions in the linear and nonlinear regimes, but are unstable to parasitic modes. Although a rigorous stability analysis of the modes in stratified, non-ideal MHD discs have not yet been done, numerical simulations suggest that they may indeed evolve into turbulence (e.g. Hawley et al. 1995; Sano et al. 2004, hereafter S04).

Having derived a criterion to identify the MRIunstable section of the $\operatorname{disc}\left(\eta \gtrsim 1\right.$ and $\left.2 \eta a^{2} \lesssim 1\right)$, we now need to quantify the turbulent angular momentum transport that we expect to develop in these regions. To accomplish this, we use the results of numerical simulations in the literature to evaluate the spatial and temporal average of the $r \phi$ component of the turbulent Maxwell stress (the main component of the stress tensor) in terms of the similarly averaged magnetic pressure, obtaining

$\ll w_{r \phi} \gg \approx 0.5 \frac{\ll B^{2} \gg}{8 \pi}$

(e.g. Hawley et al. 1995; S04). Using the characteristic ratios for the field components in MRI turbulence reported in S04, this can be expressed as

$\ll w_{r \phi} \gg \approx 14 Y \frac{B_{z, \mathrm{i}}^{2}}{8 \pi}$,

where $Y \equiv \ll B_{z}^{2} \gg / B_{z, \mathrm{i}}^{2}$ is also listed in $\mathrm{S} 04$ as a function of $\beta_{\mathrm{i}} \equiv 2 / a_{\mathrm{i}}^{2}$, the initial plasma $\beta$ parameter. Finally, the radial angular-momentum transport associated with this term is added to the angular momentum conservation equation in the disc region deemed to be unstable to the MRI. This equation then reads

$\frac{\rho v_{r} v_{K}}{2 r}+\rho v_{z} \frac{d v_{\phi}}{d z}=-\frac{J_{r} B_{z}}{c}-\frac{\ll w_{r \phi} \gg}{r}$,

where we used $(1 / r) \partial\left(r^{2} \ll w_{r \phi} \gg\right) / \partial r \approx \ll w_{r \phi} \gg$, $\partial\left(r v_{\phi}\right) / \partial r \approx v_{K} / 2$ and $J_{z} \approx 0$. Note that we are implicitly assuming that the turbulent magnetic field components only contribute to the radial angular-momentum transport and interpret the other magnetic-field (and velocity) terms in the disc structure equations as the mean values of the respective quantities. Similarly, we take the first term on the r.h.s. of equation (4) as the 
vertical angular-momentum transport induced by the mean field. The underlying assumptions of this approach would need to be verified numerically.

\section{Combined angular momentum transport}

We now explore solutions where both radial and vertical angular momentum transport take place at the same radial location in the disc. From the above discussion, it is clear that they require moderate coupling $(\eta \sim 1)$ and field strengths $\left[a_{0}<\left(2 \eta_{0}\right)^{-1 / 2}\right.$ but $\left.a\left(z=z_{\mathrm{b}}\right) \sim 1\right]$ for a wind to develop and the MRI to be active over a sizable section of the disc. To satisfy these requirements, we have allowed $\eta$ to decrease with $z$ and fall slightly below 1 in these solutions (with the expectation that it is still large enough to sustain MRI turbulence; see Sano \& Stone 2002a,b). The adopted profile (with $\eta$ decreasing from 0.63 to 0.5 between the midplane and the surface) is motivated by the inferred behavior of $\eta(z)$ in the ambipolar-diffusion dominated regions of protostellar disks (see SW07 and Salmeron \& Wardle 2005).

Fig. 2 shows solutions obtained under these assumptions. The solid lines display a pure wind (vertical angular-momentum transport only) solution. The dashed lines depict how this solution is modified when radial angular-momentum transport is incorporated via the prescription introduced in Section 3. Both solutions are compared in Table 1. Note that the addition of radial angular-momentum transport results in this case in an increased inflow speed (measured by $\epsilon$ ) and a higher mass inflow rate $\left(\dot{M}_{\text {in }}\right)$. The higher $\left|v_{r}\right|$ corresponds to a stronger radial neutral-ion drag (for $\epsilon_{\mathrm{B}}=0$; see footnote 2), and therefore to a stronger radial bending of the magnetic field lines (e.g. a higher $B_{r, \mathrm{~b}}$ ). This, in turn, increases the level of magnetic squeezing (reflected in the lower value of $h$ ), and leads to a stronger density stratification (e.g. a lower $\left.\rho_{\mathrm{s}} / \rho_{0}\right)$. Although the disc thickness does not change appreciably $\left(z_{\mathrm{b}}=1.25 h_{\mathrm{T}}\right.$ in both cases) and the height of the sonic point $\left(z_{\mathrm{s}} / h_{\mathrm{T}}\right)$ decreases (from 2.90 to 2.82 ), the lower $\rho_{\mathrm{s}} / \rho_{0}$ leads to a lower wind outflow rate and vertical torque (measured by $\kappa$ and $T_{z}$, respectively) in the combined solution.

The above results suggest that the joint operation of these two angular-momentum transport mechanisms might be self-limiting (in which case it would be unlikely to introduce a dynamical instability): The incorporation of MRI-induced turbulence increases the overall angular momentum transport in the disc, but reduce the amount of angular momentum carried away by the wind. This conclusion could be tested through an explicit stability analysis (e.g. Königl 2004) or via numerical simulations.
Table 1 Comparison of the solutions in Fig. 2. $\dot{M}_{\text {in }}$ is the mass accretion rate per disc circumference, normalized by $\left(\rho_{0} c_{\mathrm{s}} h_{\mathrm{T}}\right)$. The vertical $\left(T_{z}\right)$ and radial $\left(T_{r}\right)$ torques per unit area are normalized by $r_{0} B_{0}^{2} / 4 \pi$. All heights are listed in units of $h \equiv z\left(\rho=\rho_{0} / \sqrt{e}\right)$.

\begin{tabular}{|c|c|c|}
\hline $\begin{array}{c}\text { Disc/outflow } \\
\text { properties }\end{array}$ & $\begin{array}{c}\text { Pure wind } \\
\text { solution }\end{array}$ & $\begin{array}{c}\text { Combined } \\
\text { solution }\end{array}$ \\
\hline$h / h_{\mathrm{T}}$ & 0.31 & 0.29 \\
$z_{\mathrm{b}} / h$ & 3.99 & 4.35 \\
$z_{\mathrm{s}} / h$ & 9.25 & 9.81 \\
$\rho_{s} / \rho_{0}$ & $1.0 \times 10^{-2}$ & $8.3 \times 10^{-3}$ \\
$\kappa$ & 0.17 & 0.14 \\
$B_{r, \mathrm{~b}} / B_{z}$ & 1.75 & 1.81 \\
$\left|B_{\phi, b}\right| / B_{z}$ & 1.44 & 1.43 \\
$T_{z}$ & 1.63 & 1.59 \\
$T_{r}$ & - & 0.09 \\
$\dot{M}_{\text {in }}$ & 1.74 & 1.84 \\
\hline
\end{tabular}

\section{Discussion}

We have examined the two leading processes thought to be responsible for transporting angular momentum in protostellar discs: Jets and outflows accelerated centrifugally from the disc surfaces and turbulent viscosity induced by the magnetorotational instability. Our main motivation has been twofold. First, we have set out to explore these processes under more realistic conditions, taking into account the detailed ionisation structure and vertical stratification of the disc. Second, we are interested in the possibility that both forms of transport could operate at the same radial location and, if so, what is their relative importance at different locations and evolutionary stages in protostellar discs. Our overall formulation incorporates a height dependent, tensor conductivity. This enables us to model the different diffusion mechanisms available to the fluid (ambipolar, hall and ohmic) and how their relative contributions change with location. The illustrative examples presented here, however, assume ambipolar diffusion dominates over the entire section of the disc and the magnetic coupling is constant with height. Our main results are highlighted below.

1. We derived an approximate criterion to identify the section of the disc that would be unstable to MRI-induced turbulence and developed a scheme, based on the results of published numerical simulations, to quantify the amount of angular momentum transported radially in this region.

2. We showed a weak field solution exhibiting oscillatory field and velocity profiles and associated 

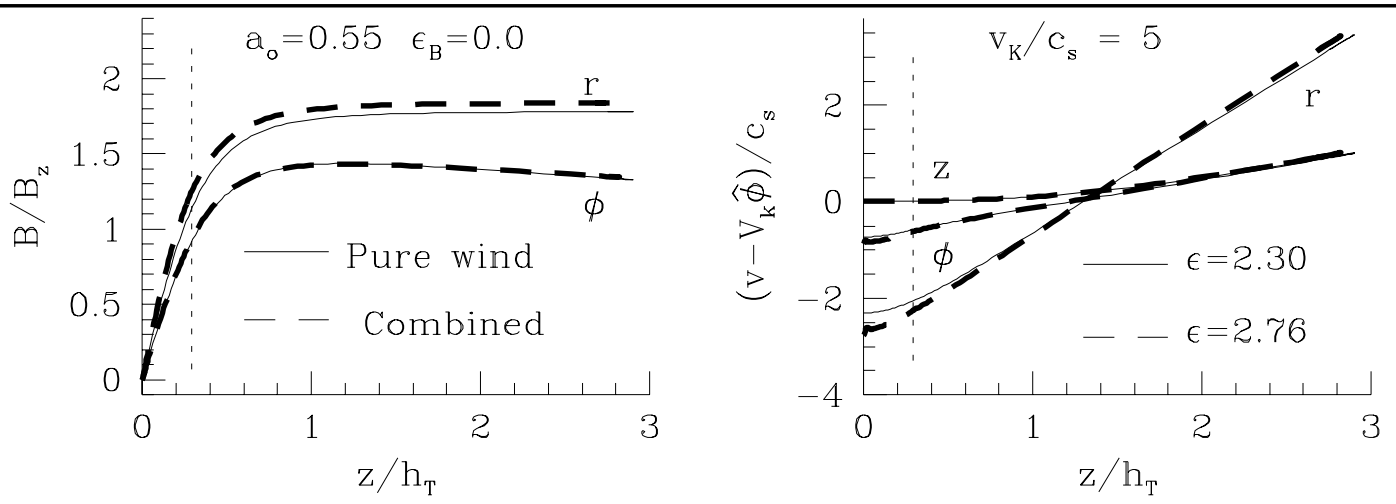

Fig. 2.- Wind-driving disc with a moderately strong field and $\eta$ decreasing from 0.63 to 0.5 between $z=0$ and $z=z_{b}$. Density, magnetic field components (left) and velocity components (right) are shown for a pure wind solution (solid lines) and also for a case where radial transport is included (dashed lines) in the region where $2 \eta a^{2}<1$, or to the left of the vertical dotted lines. The curves terminate at the respective sonic points.

it with the nonlinear MRI channel mode identified by Goodman \& Xu (1994) in an ideal-MHD, unstratified case. A rigorous stability analysis of our solutions has not been attempted yet, but evidence from linear analyses and numerical simulations suggests that they may be unstable.

3. We argued that radial angular-momentum transport operates where $2 \eta a^{2}<1$ and presented a hybrid solution in which this transition occurs at an intermediate height below the launching region.

4. This solution shows that the addition of radial transport increases the total amount of angular momentum removed from the inflowing gas, but reduces the angular momentum flux carried away by the wind.

5. It appears that these two transport mechanisms are unlikely to have a significant spatial overlap in real discs, as this requires a moderate $a_{0}(\gtrsim 0.5)$ and low (and decreasing with height) values of $\eta\left(\eta_{0} \lesssim 1\right)$ that are unlikely to be attained over measurable radial extents.

We are currently in the process of applying our general scheme to the investigation of various conductivity regimes in realistic disc models. This would enable us to estimate the fractions of angular momentum transported via turbulent viscosity and outflows, respectively; and assess the implications of the resulting disc structure to other discs processes, in particular to planet formation and migration.

\section{Acknowledgments}

We thank the referee for useful comments. This research was supported by NASA Theoretical Astrophysics Program grant NNG04G178G and the Australian Research Council.

\section{References}

Balbus S.A., Hawley J.F., 1998, Rev. Mod. Phys., 70, 1 Blandford R.D., Payne D.G., 1982, MNRAS, 199, 883 (BP82)

Casse F., Ferreira J., 2000, A\&A, 353, 1115

Casse F., Keppens R., 2002, ApJ, 581, 988

Goodman J., Xu G., 1994, ApJ, 432, 213

Hartigan P., Edwards S. Ghandour L., 1995, ApJ, 452, 736

Hawley J., Gammie C., Balbus S., 1995, ApJ, 440, 742

Königl A., 1989, ApJ, 342, 208

Königl A., 2004, ApJ, 617, 1267

Königl A., Pudritz R. E., 2000, in Mannings, V. G., Boss, A. P., Russell, S. eds, Protostars \& Planets IV. Univ. Arizona Press, Tucson, p. 759

Krasnopolsky R., Königl A., 2002, ApJ, 580, 987

Li Z.-Y. 1995, ApJ, 444, 848

Li Z.-Y. 1996, ApJ, 465, 855

Lovelace R. V. E., Romanova M. M., \& Newman W. I., 1994, ApJ, 437, 136

Ogilvie G. I., Livio M., 2001, ApJ, 553,158

Salmeron R., Königl A., Wardle M., 2007, MNRAS, 375, 177 (SKW07)

Salmeron R., Wardle M., 2003, MNRAS, 345, 992

Salmeron R., Wardle M., 2005, MNRAS, 361, 45

Sano T., Inutsuka S., 2001, ApJ, 561, L179

Sano T., Stone J. M., 2002a, ApJ, 570, 314

Sano T., Stone J. M., 2002b, ApJ, 577, 534

Sano T., Inutsuka S., Turner N., Stone J. M., 2004, ApJ, $605,321(\mathrm{~S} 04)$

Wardle M., 1999, MNRAS, 307, 849

Wardle, M., Königl, A. 1993, ApJ, 410, 218 (WK93)

This 2-column preprint was prepared with the AAS LATEX macros $\mathrm{v} 5.2$. 